Turk. J. Math. Comput. Sci.

12(2)(2020) 101-111

(C) MatDer

DOI : $10.47000 /$ tjmcs. 727448

\title{
On the New Double Binomial Sequence Space
}

\author{
Serkan Demiriz ${ }^{1, *}$ (D), Sezer Erdem ${ }^{2}$ (D) \\ ${ }^{1}$ Department of Mathematics, Faculty of Science and Arts, Tokat Gaziosmanpaşa University, 60250, Tokat, Turkey. \\ ${ }^{2}$ Battalgazi Farabi Anatolian Imam Hatip High School, 44400, Malatya, Turkey.
}

Received: 27-04-2020 • Accepted: 18-09-2020

\begin{abstract}
Aвstract. The aim of this paper is to present the new double Binomial sequence space $\mathcal{B}_{p}^{r, s}$ which consists of all sequences whose double Binomial transforms of orders $r, s$ ( $r$ and $s$ are nonzero real numbers with $r+s \neq 0$ ) are in the space $\mathcal{L}_{p}$, where $0<p<\infty$. We examine its topological and algebraic properties and inclusion relations. Furthermore, the $\alpha-, \beta(b p)-$ and $\gamma$-duals of the space $\mathcal{B}_{p}^{r, s}$ are determined and finally, some 4-dimensional matrix mapping classes related to this space are characterized.
\end{abstract}

2010 AMS Classification: 46A06, 47B38

Keywords: Double sequence spaces, Binomial matrix, $\alpha-, \beta(b p)$ - and $\gamma$-duals, Matrix domain of 4-dimensional matrices, Matrix transformations.

\section{INTRODUCTION}

Let us begin by giving some basic concepts which are going to be used in the rest of the article. All complex valued double sequences are represented by $\Omega$ which is a vector space with coordinatewise addition and scalar multiplication. Any linear subspace of $\Omega$ is known as double sequence space. The set of all bounded complex valued double sequences is denoted by $\mathcal{M}_{u}$, that is,

$$
\mathcal{M}_{u}=\left\{u=\left(u_{i j}\right) \in \Omega:\|u\|_{\infty}=\sup _{i, j \in \mathbb{N}}\left|u_{i j}\right|<\infty\right\},
$$

where $\mathbb{N}=\{0,1,2, \ldots\}$. We say that the double sequence $u=\left(u_{i j}\right)$ is convergent in the Pringsheim's sense if for every $\varepsilon>0$ there exists $n_{\varepsilon} \in \mathbb{N}$ such that $\left|u_{i j}-L\right|<\varepsilon$ whenever $i, j>n_{\varepsilon} . L \in \mathbb{C}$ is called the Pringsheim limit of $u$ and stated by $p-\lim _{i, j \rightarrow \infty} u_{i j}=L$; where $\mathbb{C}$ denotes the complex field. $C_{p}$ represents the space of all such $u$ which are called shortly as $p$-convergent. Of particular interest is unlike single sequences, $p$-convergent double sequences need not be bounded. For example, consider the sequence $u=\left(u_{i j}\right)$ identified by

$$
u_{i j}:=\left\{\begin{array}{ccc}
i & , & i \in \mathbb{N}, j=0, \\
j & , & j \in \mathbb{N}, i=0, \\
0 & , & i, j \in \mathbb{N} \backslash\{0\} .
\end{array}\right.
$$

Then, it can be easily seen that $p-\lim u_{i j}=0$ but $\sup _{i, j \in \mathbb{N}}\left|u_{i j}\right|=\infty$. As a conclusion $u \in C_{p} \backslash \mathcal{M}_{u}$. The bounded sequences which are also $p$-convergent are indicated by $\mathcal{C}_{b p}$, that is, $C_{b p}=\mathcal{C}_{p} \cap \mathcal{M}_{u}$. A double sequence $u=\left(u_{i j}\right) \in C_{p}$ is called as regularly convergent if the limits $u_{i}:=\lim _{j} u_{i j},(i \in \mathbb{N})$ and $u_{j}:=\lim _{i} u_{i j},(j \in \mathbb{N})$ exist, and the limits

*Corresponding Author

Email addresses: serkandemiriz@gmail.com (S. Demiriz), sezererdem8344@gmail.com (S. Erdem) 
$\lim _{i} \lim _{j} u_{i j}$ and $\lim _{j} \lim _{i} u_{i j}$ exist and are equivalent to the $p-\lim$ of $u$. The space of all such double sequences is denoted by $C_{r}$. Obviously, the regular convergence of a double sequence $u$ implies the convergence in Pringsheim's sense as well as the boundedness of the terms of $u$, but the converse implication fails. A sequence $u=\left(u_{i j}\right)$ is called double null sequence if it converges to zero. Additionally, all double null sequences in the spaces $C_{b p}$ and $C_{r}$ are represent by $C_{b p 0}$ and $C_{r 0}$, respectively. Móricz [10] showed that the spaces $\mathcal{M}_{u}, C_{b p}, C_{b p 0}, C_{r}$ and $C_{r 0}$ are Banach spaces endowed with the norm $\|.\|_{\infty}$.

Let us take any $u \in \Omega$ and consider the sequence $S=\left(s_{k l}\right)$ defined by

$$
s_{k l}:=\sum_{i=0}^{k} \sum_{j=0}^{l} u_{i j}, \quad(k, l \in \mathbb{N}) .
$$

Thus, the pair $\left(\left(u_{k l}\right),\left(s_{k l}\right)\right)$ is called as double series. Here, the sequence $S=\left(s_{k l}\right)$ is the sequence of partial sums of the double series.

Let $\Psi$ be a space of double sequences, converging with respect to some linear convergence rule $\vartheta-\lim : \Psi \rightarrow \mathbb{C}$. The sum of a double series $\sum_{i, j} u_{i j}$ with respect to this rule is defined by $\vartheta-\sum_{i, j} u_{i j}=\vartheta-\lim _{k, l \rightarrow \infty} \sum_{i, j}^{k, l} u_{i j}$. Here and thereafter, we will use the summation $\sum_{i, j}$ instead of $\sum_{i=0}^{\infty} \sum_{j=0}^{\infty}$ and assume $\vartheta \in\{p, b p, r\}$. In the rest of the article $p^{\prime}$ denotes the conjugate of $p$, that is, $p^{\prime}=p /(p-1)$ for $1<p<\infty$.

The $\alpha$-dual $\Psi^{\alpha}, \beta(\vartheta)$-dual $\Psi^{\beta(\vartheta)}$ with respect to the $\vartheta$-convergence and the $\gamma-d u a l \Psi^{\gamma}$ of a double sequence space $\Psi$ are respectively described as

$$
\begin{aligned}
\Psi^{\alpha} & :=\left\{t=\left(t_{i j}\right) \in \Omega: \sum_{i, j}\left|t_{i j} u_{i j}\right|<\infty \quad \text { for all } \quad\left(u_{i j}\right) \in \Psi\right\}, \\
\Psi^{\beta(\vartheta)} & :=\left\{t=\left(t_{i j}\right) \in \Omega: \vartheta-\sum_{i, j} t_{i j} u_{i j} \quad \text { exists for all } \quad\left(u_{i j}\right) \in \Psi\right\}, \\
\Psi^{\gamma} & :=\left\{t=\left(t_{i j}\right) \in \Omega: \sup _{k, l \in \mathbb{N}}\left|\sum_{i, j=0}^{k, l} t_{i j} u_{i j}\right|<\infty \quad \text { for all } \quad\left(u_{i j}\right) \in \Psi\right\} .
\end{aligned}
$$

It can be easily seen that if $\Psi \subset \Lambda$, then $\Lambda^{\alpha} \subset \Psi^{\alpha}$. Furthermore, $\Psi^{\alpha} \subset \Psi^{\gamma}$ for the double sequence spaces $\Psi$ and $\Lambda$.

Now, we shall deal with matrix mapping. Let us consider double sequence spaces $\Psi$ and $\Lambda$ and the 4-dimensional complex infinite matrix $D=\left(d_{k l i j}\right)$. Then, it is said that $D$ is a matrix mapping from $\Psi$ into $\Lambda$ and is written as $D: \Psi \rightarrow \Lambda$, if for every $u=\left(u_{i j}\right) \in \Psi$, the $D$-transform $D u=\left\{(D u)_{k l}\right\}_{k, l \in \mathbb{N}}$ of $u$ exists and is in $\Lambda$, where

$$
(D u)_{k l}=\vartheta-\sum_{i, j} d_{k l i j} u_{i j} \quad \text { for each } \quad k, l \in \mathbb{N} .
$$

$(\Psi: \Lambda)$ stands for the class of all 4-dimensional complex infinite matrices from a double sequence space $\Psi$ into a double sequence space $\Lambda$. Then, $D \in(\Psi: \Lambda)$ if and only if $D_{k l} \in \Psi^{\beta(\vartheta)}$, where $D_{k l}=\left(d_{k l i j}\right)_{i, j \in \mathbb{N}}$ for all $k, l \in \mathbb{N}$.

The $\vartheta$-summability domain $\Psi_{D}^{(\vartheta)}$ of a 4-dimensional complex infinite matrix $D$ in a double sequence space $\Psi$ consists of whose $D$-transforms are in $\Psi$; that is,

$$
\Psi_{D}^{(\vartheta)}:=\left\{u=\left(u_{i j}\right) \in \Omega: D u:=\left(\vartheta-\sum_{i j} d_{k l i j} u_{i j}\right)_{k, l \in \mathbb{N}} \text { exists and is in } \Psi\right\} .
$$

In the past, double sequence spaces have been studied by many authors. Now, let us give some information about these studies. In her doctoral dissertation, Zeltser [21] has fundamentally examined both the topological structure and the theory of summability of double sequences. Recently, Altay and Başar [1] defined the double sequence spaces $\mathcal{B S}$, $\mathcal{B S}(t), C \mathcal{S}_{p}, C \mathcal{S}_{b p}, C \mathcal{S}_{r}$ and $\mathcal{B V}$ of double series whose sequences of partial sums are in the spaces $\mathcal{M}_{u}, \mathcal{M}_{u}(t), C_{p}$, $C_{b p}, C_{r}$ and $\mathcal{L}_{u}$, respectively, and also examined some properties of those sequence spaces. Later, in [3], Başar and Sever have defined the Banach space $\mathcal{L}_{p}$ by

$$
\mathcal{L}_{p}:=\left\{\left(u_{i j}\right) \in \Omega: \sum_{i, j}\left|u_{i j}\right|^{p}<\infty\right\}, \quad(1 \leq p<\infty)
$$


with the norm $\|\cdot\|_{\mathcal{L}_{p}}$, which is defined in the following way:

$$
\|.\|_{\mathcal{L}_{p}}=\left(\sum_{i, j}\left|u_{i j}\right|^{p}\right)^{\frac{1}{p}} .
$$

It is also significant that the double sequence space $\mathcal{L}_{u}$ which was defined by Zeltser [22] is the special case of the space $\mathcal{L}_{p}$ for $p=1$. For more details about the double sequences and related topics, the reader may refer to Altay and Bașar [1], Bașar [2], Bașar and Sever [3], Çapan [6], Demiriz and Duyar [7], Demiriz and Erdem [8], Mursaleen [11], Talebi [14], Tug̃ [15-17] and Yeșilkayagil and Bașar [18-20].

The sequence space $\mathcal{E}_{p}^{r, s}$ has been studied by Talebi [14] for $1 \leq p<\infty$ and also by Yeșilkayagil and Bașar [20] for $0<p<1$ as the set of all double sequences such that $E(r, s)$-transforms of them are in the spaces $\mathcal{L}_{p}$ where $E(r, s)$ denotes the method of double Euler means of orders $r, s(0<r, s<1)$ defined by the 4-dimensional matrix $E(r, s)=\left(e_{k l i j}^{r, s}\right)$, which is described in the following way:

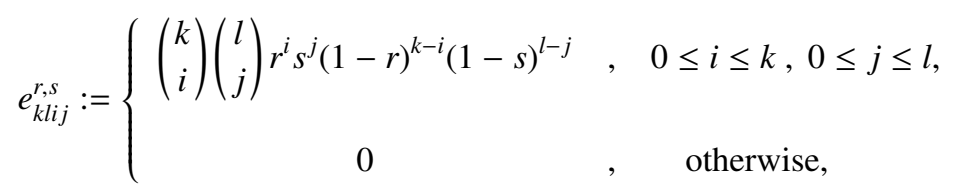

for every $k, l, i, j \in \mathbb{N}$.

Assume that $r, s$ and $r+s$ are nonzero real numbers. Then, the 2-dimensional binomial matrix $B^{r, s}=\left(b_{k i}^{r, s}\right)$ is described in the following way:

$$
b_{k i}^{r, s}:=\left\{\begin{array}{cc}
\frac{1}{(r+s)^{k}}\left(\begin{array}{c}
k \\
i
\end{array}\right) s^{k-i} r^{i}, & 0 \leq i \leq k, \\
0 & \text { otherwise, }
\end{array}\right.
$$

for every $k, i \in \mathbb{N}$. It should be noted that if $r . s>0$, then the 2-dimensional binomial matrix is regular. By using the binomial matrix $B^{r, s}=\left(b_{k i}^{r, s}\right)$, the binomial sequence spaces $b_{p}^{r, s}$ and $b_{\infty}^{r, s}$ which consist of all sequences whose $B^{r, s}$-transforms are in the spaces $\ell_{p}$ of absolutely $p$-summable and $\ell_{\infty}$ of bounded single sequences are introduced by Bisgin. For more details on the spaces $b_{p}^{r, s}$ and $b_{\infty}^{r, s}$, the reader may refer to [4].

The general frame of the rest of the study can be given as follows: In the second section, at the beginning the double sequence space $\mathcal{B}_{p}^{r, s}$ is introduced, where $0<p<\infty$. Also, the algebraic and topological properties of this space are examined and some inclusion relations are given. In section 3, we determine the $\alpha-, \beta(b p)-$ and $\gamma$-duals of the space $\mathcal{B}_{p}^{r, s}$. Finally, in the last section, some matrix classes on this new space are characterized.

\section{Main Results}

In the current section, we present the double sequence space $\mathcal{B}_{p}^{r, s}$ by using the 4-dimensional Binomial mean $B^{(r, s)}$ of orders $r, s$ and give some properties and results on this space.

Assume that $r, s$ and $r+s$ are nonzero real numbers. Now, we define the binomial mean of orders $r$ and $s$ for double sequences described by the 4-dimensional matrix $B^{(r, s)}=\left(b_{k l i j}^{r, s}\right)$ as follows:

$$
b_{k l i j}^{r, s}:=\left\{\begin{array}{cl}
\frac{1}{(r+s)^{k+l}}\left(\begin{array}{c}
k \\
i
\end{array}\right)\left(\begin{array}{l}
l \\
j
\end{array}\right) s^{k+j-i} r^{l+i-j}, & 0 \leq i \leq k, 0 \leq j \leq l, \\
0 & \text { otherwise, }
\end{array}\right.
$$

for every $k, l, i, j \in \mathbb{N}$. Therefore, the $B^{(r, s)}$-transform of a double sequence $u=\left(u_{i j}\right)$ is given by

$$
v_{k l}:=\left\{B^{(r, s)} u\right\}_{k l}=\sum_{i, j}^{k, l} \frac{1}{(r+s)^{k+l}}\left(\begin{array}{c}
k \\
i
\end{array}\right)\left(\begin{array}{l}
l \\
j
\end{array}\right) s^{k+j-i} r^{l+i-j} u_{i j},
$$

for every $k, l \in \mathbb{N}$. We will assume unless stated otherwise that the double sequences $u=\left(u_{i j}\right)$ and $v=\left(v_{i j}\right)$ are connected with the relation (2.2) and $r, s$ and $r+s$ are nonzero real numbers. We would like touch on a point, in the special case $r+s=1$, we obtain the 4-dimensional Euler matrix $E(r, s)=\left(e_{k l i j}^{r, s}\right)$ from the 4-dimensional Binomial 
matrix $B^{(r, s)}=\left(b_{k l i j}^{r, s}\right)$. So, the matrix $B^{(r, s)}$ generalizes the matrix $E(r, s)$. Let $I=\left(\delta_{k l i j}\right)$ be a 4-dimensional unit matrix, that is,

$$
\delta_{k l i j}=\left\{\begin{array}{cc}
1, & (k, l)=(i, j) \\
0, & \text { otherwise }
\end{array}\right.
$$

It is clear that by using the equality

$$
\delta_{k l i j}=\sum_{m, n} b_{k l m n}^{r, s} \cdot c_{m n i j}^{r, s},
$$

the inverse $\left\{B^{(r, s)}\right\}^{-1}=\left(c_{k l i j}^{r, s}\right)$ of the triangle matrix $B^{(r, s)}$ can be found as

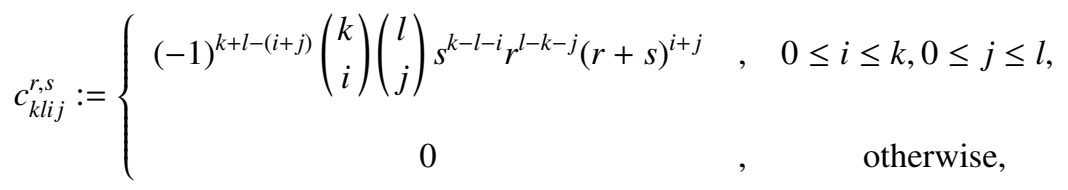

for every $k, l, i, j \in \mathbb{N}$. We introduce the sequence space $\mathcal{B}_{p}^{r, s}$ as the set of all double sequences such that $B^{(r, s)}$-transforms of them are in the space $\mathcal{L}_{p}$, that is,

$$
\mathcal{B}_{p}^{r, s}=\left\{u=\left(u_{i j}\right) \in \Omega: \sum_{k, l}\left|\sum_{i, j}^{k, l} \frac{1}{(r+s)^{k+l}}\left(\begin{array}{l}
k \\
i
\end{array}\right)\left(\begin{array}{l}
l \\
j
\end{array}\right) s^{k+j-i} r^{l+i-j} u_{i j}\right|^{p}<\infty\right\}
$$

for $0<p<\infty$. In that case, $\mathcal{B}_{p}^{r, s}$ can be rewritten as $\mathcal{B}_{p}^{r, s}=\left(\mathcal{L}_{p}\right)_{B^{(r, s)}}$ with the notation of (1.1). If $\Psi$ is any normed double sequence space, then we call the matrix domain $\Psi_{B^{(r, s)}}$ as the double Binomial sequence space.

Definition 2.1 (See [9, 12]). A 4-dimensional matrix D is said to be RH-regular if it maps every bounded $p$-convergent sequence into a $p$-convergent sequence with the same $p$-limit.

Lemma 2.2 (See $[9,12])$. A 4-dimensional triangle matrix $D=\left(d_{k l i j}\right)$ is $R H$-regular if and only if

$$
\begin{aligned}
& R H_{1}: p-\lim _{k, l \rightarrow \infty} d_{k l i j}=0 \quad \text { for each } i, j \in \mathbb{N}, \\
& R H_{2}: p-\lim _{k, l \rightarrow \infty} \sum_{i, j} d_{k l i j}=1, \\
& R H_{3}: p-\lim _{k, l \rightarrow \infty} \sum_{i}\left|d_{k l i j}\right|=0 \text { for each } j \in \mathbb{N}, \\
& R H_{4}: p-\lim _{k, l \rightarrow \infty} \sum_{j}\left|d_{k l i j}\right|=0 \text { for each } i \in \mathbb{N},
\end{aligned}
$$

$\mathrm{RH}_{5} \quad$ : $\quad$ There exists finite positive integers $M$ and $N$ such that

$$
\sum_{i, j>N}\left|d_{k l i j}\right|<M
$$

Theorem 2.3. Let r.s $>0$. Then the 4-dimensional Binomial matrix $B^{(r, s)}$ of orders $r$, $s$ defined by (2.1) is RH-regular.

Proof. Since $\frac{1}{(r+s)^{k}}\left(\begin{array}{c}k \\ i\end{array}\right) s^{k-i} r^{i} \rightarrow 0$, as $k \rightarrow \infty$ and $\frac{1}{(r+s)^{l}}\left(\begin{array}{c}l \\ j\end{array}\right) r^{l-j} s^{j} \rightarrow 0$, as $l \rightarrow \infty$ for $r . s>0$, then it can be easily seen that $b_{k l i j}^{r, s} \rightarrow 0$, as $k, l \rightarrow \infty$ for each $i, j \in \mathbb{N}$, that is, $R H_{1}$ satisfies. By taking into account the equality

$$
\sum_{i, j} b_{k l i j}^{r, s}=\frac{1}{(r+s)^{k+l}} \sum_{i, j=0}^{k, l}\left(\begin{array}{l}
k \\
i
\end{array}\right)\left(\begin{array}{l}
l \\
j
\end{array}\right) s^{k+j-i} r^{l+i-j}=1,
$$

so, $R H_{2}$ holds. We deduce from the equation $\sum_{i}\left|b_{k l i j}\right|=\frac{1}{(r+s)^{k}}\left(\begin{array}{l}l \\ j\end{array}\right) r^{l-j} s^{j}$ that $R H_{3}$ satisfies. With the similar way, the condition $\mathrm{RH}_{4}$ holds. Using the relation (2.3) and the positivity of the matrix $B^{(r, s)}$ for $r . s>0$, i.e., $b_{k l i j}^{r, s} \geq 0$ for every $k, l, i, j \in \mathbb{N}$, it is clear that the condition $\mathrm{RH}_{5}$ satisfies. This step concludes the proof.

In the rest of the article, it will be assumed that $r . s>0$. 
Theorem 2.4. The set $\mathcal{B}_{p}^{r, s}$ becomes a linear space with coordinatewise addition and scalar multiplication for double sequences and the following statements hold:

(i) If $0<p<1$, then $\mathcal{B}_{p}^{r, s}$ is a complete $p$-normed space with

$$
\|u\|_{\mathcal{B}_{p}^{r, s}}^{2}=\left\|B^{(r, s)} u\right\|_{\mathcal{L}_{p}}^{2}=\sum_{k, l}\left|\sum_{i, j}^{k, l} \frac{1}{(r+s)^{k+l}}\left(\begin{array}{c}
k \\
i
\end{array}\right)\left(\begin{array}{l}
l \\
j
\end{array}\right) s^{k+j-i} r^{l+i-j} u_{i j}\right|^{p},
$$

which is p-norm isomorphic to the space $\mathcal{L}_{p}$.

(ii) If $1 \leq p<\infty$, then $\mathcal{B}_{p}^{r, s}$ is a Banach space with

$$
\|u\|_{\mathcal{B}_{p}^{r, s}}=\left\|B^{(r, s)} u\right\|_{\mathcal{L}_{p}}=\left(\sum_{k, l}\left|\sum_{i, j}^{k, l} \frac{1}{(r+s)^{k+l}}\left(\begin{array}{c}
k \\
i
\end{array}\right)\left(\begin{array}{l}
l \\
j
\end{array}\right) s^{k+j-i} r^{l+i-j} u_{i j}\right|^{p}\right)^{\frac{1}{p}},
$$

which is norm isomorphic to the space $\mathcal{L}_{p}$.

Proof. Part (i) can be proved in a similar way, therefore we give the proof only for part (ii).

Since the initial assertion is simple and easy to prove, we ignore its proof in here. To confirm the fact that $\mathcal{B}_{p}^{r, s}$ is norm isomorphic to the space $\mathcal{L}_{p}$, we need to be sure the existence of a linear and norm preserving bijection between the spaces $\mathcal{B}_{p}^{r, s}$ and $\mathcal{L}_{p}$ for $1 \leq p<\infty$. For this purpose, let us take the transformation $T$ defined from $\mathcal{B}_{p}^{r, s}$ into $\mathcal{L}_{p}$ by $u \mapsto v=T u$, where $v=\left(v_{k l}\right)$ is the $B^{(r, s)}$-transform of the sequence $u=\left(u_{k l}\right)$. The linearity of $T$ is clear. Consider the equality

$$
T u=\left[\begin{array}{cccc}
u_{00} & \vdots & \sum_{j=0}^{l} \frac{1}{(r+s)^{l}}\left(\begin{array}{c}
l \\
j
\end{array}\right) s^{j} r^{l-j} u_{0 j} & \vdots \\
\frac{s u_{00}+r u_{10}}{r+s} & \vdots & \sum_{i=0, j=0}^{1, l} \frac{1}{(r+s)^{1+l}}\left(\begin{array}{c}
1 \\
i
\end{array}\right)\left(\begin{array}{c}
l \\
j
\end{array}\right) s^{1+j-i} r^{l+i-j} u_{i j} & \vdots \\
\vdots & \vdots & \vdots & \vdots \\
\sum_{i=0}^{k} \frac{1}{(r+s)^{k}}\left(\begin{array}{c}
k \\
i
\end{array}\right) s^{k-i} r^{i} u_{i j} & \vdots & \sum_{i=0, j=0}^{k, l} \frac{1}{(r+s)^{k+l}}\left(\begin{array}{c}
k \\
i
\end{array}\right)\left(\begin{array}{l}
l \\
j
\end{array}\right) s^{k+j-i} r^{l+i-j} u_{i j} & \vdots \\
\vdots & \vdots & \vdots & \vdots
\end{array}\right]=\theta
$$

which yields $u_{i j}=0$ for every $i, j \in \mathbb{N}$. So, $u=\theta$. Therefore, $T$ is injective. Let us consider $v \in \mathcal{L}_{p}$ for $1 \leq p<\infty$ and describe the double sequence $u=\left(u_{k l}\right)$ by

$$
u_{k l}=\sum_{i, j}^{k, l}\left(\begin{array}{l}
k \\
i
\end{array}\right)\left(\begin{array}{l}
l \\
j
\end{array}\right)(-1)^{k+l-(i+j)} s^{k-l-i} r^{l-k-j}(r+s)^{i+j} v_{i j}
$$

for every $k, l \in \mathbb{N}$. In that case, for $1 \leq p<\infty$, it is seen that

$$
\begin{aligned}
\|u\|_{\mathcal{B}_{p}^{r, s}} & =\left\|B^{(r, s)} u\right\|_{\mathcal{L}_{p}} \\
& =\left(\sum_{k, l}\left|\sum_{i, j}^{k, l} \frac{1}{(r+s)^{k+l}}\left(\begin{array}{c}
k \\
i
\end{array}\right)\left(\begin{array}{l}
l \\
j
\end{array}\right) s^{k+j-i} r^{l+i-j} u_{i j}\right|^{p}\right)^{1 / p} \\
& =\left(\sum_{k, l}\left|\sum_{i, j}^{k, l} \delta_{k l i j} v_{i j}\right|^{p}\right)^{1 / p} \\
& =\left(\sum_{k, l}\left|v_{k l}\right|^{p}\right)^{1 / p}=\|v\|_{\mathcal{L}_{p}}<\infty .
\end{aligned}
$$

Thus, we have that $u \in \mathcal{B}_{p}^{r, s}$ for $1 \leq p<\infty$ and consequently $T$ is surjective and norm preserving. Hence, $T$ is a linear and norm preserving bijection which means that the spaces $\mathcal{B}_{p}^{r, s}$ and $\mathcal{L}_{p}$ are norm isomorphic, as desired.

Now, let us prove that $\mathcal{B}_{p}^{r, s}$ is a Banach space with the norm $\|.\|_{\mathcal{B}_{p}^{r, s}}$ described by (2.4). To do this, it can be used Section (b) of Corollary 6.3.41 in [5] which says that "Let $(X, \sigma)$ and $(Y, \varsigma)$ be semi-normed spaces and $T:(X, \sigma) \rightarrow(Y, \varsigma)$ be an isometric isomorphism. Then, $(X, \sigma)$ is complete if and only if $(Y, \varsigma)$ is complete. In particular, $(X, \sigma)$ is a Banach 
space if and only if $(Y, \varsigma)$ is a Banach space." Since the map $T$ described in the proof of this theorem from $\mathcal{B}_{p}^{r, s}$ to $\mathcal{L}_{p}$ is an isometric isomorphism and the double sequence space $\mathcal{L}_{p}$ is a Banach space from Theorem 2.1 in [3], it can be obviously seen that the space $\mathcal{B}_{p}^{r, s}$ is a Banach space. In fact, this is exactly what we want to prove.

It can be easily checked that the absolute property does not hold on the space $\mathcal{B}_{p}^{r, s}$, that is $\|u\|_{\mathcal{B}_{p}^{r, s}} \neq\|u\| \|_{\mathcal{B}_{p}^{r, s}}$ for at least one sequence in the space $\mathcal{B}_{p}^{r, s}$, and this says that $\mathcal{B}_{p}^{r, s}$ is a sequence space of nonabsolute type, where $|u|=\left(\left|u_{i j}\right|\right)$.

Definition 2.5 ( [5]). Let $\Psi$ be a locally convex space. Then, a subset of $\Psi$ is called barrel if it is absolutely convex, absorbing and closed in $\Psi$. Moreover, $\Psi$ is called a barrelled space if each barrel is a neighborhood of zero.

Lemma 2.6 ([13]). If the sequence space $\Psi$ is a Banach space or a Fréchet space, then it is a barelled space.

Theorem 2.7. Let $1 \leq p<\infty$. Then, $\mathcal{B}_{p}^{r, s}$ is a barelled space.

Proof. The proof is clearly seen by applying Theorem 2.4 and Lemma 2.6.

With the notation Zeltzer [21], we define the double sequence $e^{i j}=\left(e_{k l}^{i j}\right)$ by

$$
e_{k l}^{i j}=\left\{\begin{array}{cc}
1, & (k, l)=(i, j), \\
0, & \text { otherwise },
\end{array}\right.
$$

for every $i, j, k, l \in \mathbb{N}$.

Definition 2.8 ( [5]). A non-empty subset $X$ of a locally convex space $\Psi$ is called fundamental if the closure of the linear span of $X$ equals $\Psi$.

From the previous description, Yeşilkayagil and Başar [18] have showed that $X$ is the fundamental set of $\mathcal{L}_{p}$, where $X:=\left\{e^{i j}: i, j \in \mathbb{N}\right\}$. In the light of this fact, let us describe the double sequences $f^{i j}=\left(f_{k l}^{i j}\right)$ by

$$
f_{k l}^{i j}:=\left\{\begin{array}{cc}
(-1)^{k+l-(i+j)}\left(\begin{array}{c}
k \\
i
\end{array}\right)\left(\begin{array}{l}
l \\
j
\end{array}\right) s^{k-l-i} r^{l-k-j}(r+s)^{i+j} & , 0 \leq i \leq k, 0 \leq j \leq l, \\
0 & ,
\end{array}\right.
$$

for every $i, j, k, l \in \mathbb{N}$. Thus, $\left\{f^{i j}: i, j \in \mathbb{N}\right\}$ is the fundamental set of the space $\mathcal{B}_{p}^{r, s}$; because $B^{(r, s)} f^{i j}=e^{i j}$.

If $u t=\left(u_{i j} t_{i j}\right) \in \Psi$ for every $u=\left(u_{i j}\right) \in \Psi$ and $t=\left(t_{i j}\right) \in\{0,1\}^{\mathbb{N} \times \mathbb{N}}$, then we say that a double sequence space $\Psi$ is monotone, where $\{0,1\}^{\mathbb{N} \times \mathbb{N}}$ represents the set of all double sequences of zeros and ones.

Theorem 2.9. Let $1 \leq p<\infty$. If $\left(\frac{s^{k} r^{l}}{(r+s)^{k+l}}\right) \notin \mathcal{L}_{p}$, then the space $\mathcal{B}_{p}^{r, s}$ is not monotone.

Proof. Let $1 \leq p<\infty$ and $\left(\frac{s^{k} r^{l}}{(r+s)^{k+l}}\right) \notin \mathcal{L}_{p}$. Let us select the sequence $u=\left(u_{i j}\right) \in \mathcal{B}_{p}^{r, s}$ as $u_{00} \neq 0$ and define the sequence $t=\left(t_{i j}\right)=e^{00} \in\{0,1\}^{\mathbb{N} \times \mathbb{N}}$. Thus, if we take the sequence $z=t u=e^{00} u$, it can be easily seen that

$$
\left(B^{(r, s)} z\right)_{k l}=\frac{s^{k} r^{l}}{(r+s)^{k+l}} u_{00} .
$$

Since $\left(\frac{s^{k} r^{l}}{(r+s)^{k+l}}\right) \notin \mathcal{L}_{p}$, then $\left(B^{(r, s)} z\right) \notin \mathcal{L}_{p}$. Therefore, $z \notin \mathcal{B}_{p}^{r, s}$. This completes the proof.

Theorem 2.10. The inclusion $\mathcal{L}_{p} \subset \mathcal{B}_{p}^{r, s}$ strictly holds for $1 \leq p<\infty$. 
Proof. Suppose that $u=\left(u_{i j}\right) \in \mathcal{L}_{p}$ is an arbitrary double sequence. By applying Hölder's inequality together taking into account the relation (2.2), we obtain that

$$
\begin{aligned}
\left|v_{k l}\right|^{p} & =\left|\sum_{i, j}^{k, l} \frac{1}{(r+s)^{k+l}}\left(\begin{array}{c}
k \\
i
\end{array}\right)\left(\begin{array}{l}
l \\
j
\end{array}\right) s^{k+j-i} r^{l+i-j} u_{i j}\right|^{p} \\
& \leq\left(\sum_{i, j}^{k, l} \frac{1}{(r+s)^{k+l}}\left(\begin{array}{l}
k \\
i
\end{array}\right)\left(\begin{array}{l}
l \\
j
\end{array}\right) s^{k+j-i} r^{l+i-j}\left|u_{i j}\right|^{p}\right) \\
& \times\left(\sum_{i, j}^{k, l} \frac{1}{(r+s)^{k+l}}\left(\begin{array}{l}
k \\
i
\end{array}\right)\left(\begin{array}{l}
l \\
j
\end{array}\right) s^{k+j-i} r^{l+i-j}\right)^{p-1} \\
& =\left(\sum_{i, j}^{k, l} \frac{1}{(r+s)^{k+l}}\left(\begin{array}{l}
k \\
i
\end{array}\right)\left(\begin{array}{l}
l \\
j
\end{array}\right) s^{k+j-i} r^{l+i-j}\left|u_{i j}\right|^{p}\right) \\
& \times\left(\frac{1}{(r+s)^{k}} \sum_{i=0}^{k}\left(\begin{array}{c}
k \\
i
\end{array}\right) s^{k-i} r^{i} \frac{1}{(r+s)^{l}} \sum_{j=0}^{l}\left(\begin{array}{l}
l \\
j
\end{array}\right) s^{j} r^{l-j}\right)^{p-1} \\
& =\sum_{i, j}^{k, l} \frac{1}{(r+s)^{k+l}}\left(\begin{array}{c}
k \\
i
\end{array}\right)\left(\begin{array}{l}
l \\
j
\end{array}\right) s^{k+j-i} r^{l+i-j}\left|u_{i j}\right|^{p} .
\end{aligned}
$$

By applying (2.6), it is seen that

$$
\begin{aligned}
\sum_{k, l}\left|v_{k l}\right|^{p} & \leq \sum_{k, l}\left(\sum_{i, j}^{k, l} \frac{1}{(r+s)^{k+l}}\left(\begin{array}{l}
k \\
i
\end{array}\right)\left(\begin{array}{l}
l \\
j
\end{array}\right) s^{k+j-i} r^{l+i-j}\left|u_{i j}\right|^{p}\right) \\
& =\sum_{i, j}^{\infty, \infty}\left|u_{i j}\right|^{p}\left(\sum_{k=i}^{\infty} \sum_{l=j}^{\infty} \frac{1}{(r+s)^{k+l}}\left(\begin{array}{c}
k \\
i
\end{array}\right)\left(\begin{array}{l}
l \\
j
\end{array}\right) s^{k+j-i} r^{l+i-j}\right) \\
& =\sum_{i, j}^{\infty, \infty}\left|u_{i j}\right|^{p}\left(\sum_{k=i}^{\infty} \frac{1}{(r+s)^{k}}\left(\begin{array}{l}
k \\
i
\end{array}\right) s^{k-i} r^{i} \sum_{l=j}^{\infty} \frac{1}{(r+s)^{l}}\left(\begin{array}{l}
l \\
j
\end{array}\right) r^{l-j} s^{j}\right) \\
& =\sum_{i, j}^{\infty, \infty}\left|u_{i j}\right|^{p}\left(\sum_{k=i}^{\infty}\left(\begin{array}{c}
k \\
i
\end{array}\right)\left(\frac{s}{r+s}\right)^{k}\left(\frac{r}{s}\right)^{i} \sum_{l=j}^{\infty}\left(\begin{array}{l}
l \\
j
\end{array}\right)\left(\frac{r}{r+s}\right)^{l}\left(\frac{s}{r}\right)^{j}\right) \\
& =\frac{(r+s)^{2}}{r s} \sum_{i, j}^{\infty, \infty}\left|u_{i j}\right|^{p}
\end{aligned}
$$

which yields us that

$$
\|u\|_{\mathcal{B}_{p}^{r, s}} \leq\left(\frac{(r+s)^{2}}{r s}\right)^{1 / p}\|u\|_{\mathcal{L}_{p}}
$$

Moreover, since the sequence $u=\left(u_{i j}\right)=(-1)^{i+j}$ is in $\mathcal{B}_{p}^{r, s}-\mathcal{L}_{p}$, then the inclusion $\mathcal{L}_{p} \subset \mathcal{B}_{p}^{r, s}$ is strict for $1 \leq p<\infty$.

Theorem 2.11. Let $1 \leq p<p_{1}<\infty$. Then the inclusion $\mathcal{B}_{p}^{r, s} \subset \mathcal{B}_{p_{1}}^{r, s}$ holds.

Proof. Suppose that $u=\left(u_{i j}\right) \in \mathcal{B}_{p}^{r, s}$ is an arbitrary double sequence. Then, $B^{(r, s)} u \in \mathcal{L}_{p}$. Since the inclusion $\mathcal{L}_{p} \subset \mathcal{L}_{p_{1}}$ for $1 \leq p<p_{1}<\infty$ from Bașar and Sever [3], it is concluded that $B^{(r, s)} u \in \mathcal{L}_{p_{1}}$. Hence $u \in \mathcal{B}_{p_{1}}^{r, s}$, as desired.

$$
\text { 3. The } \alpha-, \beta(b p)-\text { AND } \gamma \text {-Duals of The Space } \mathcal{B}_{p}^{r, s}
$$

In the present section, we will determine the $\alpha-, \beta(b p)-$ and $\gamma$-duals of the space $\mathcal{B}_{p}^{r, s}$. For this purpose, firstly we need to give a lemma. 
Lemma 3.1 ( [18]). Suppose that $D=\left(d_{k l i j}\right)$ be a 4-dimensional infinite matrix. At that time, the following statements hold:

(i) Assume that $0<p \leq 1$. In that case, $D \in\left(\mathcal{L}_{p}: \mathcal{M}_{u}\right)$ iff

$$
N=\sup _{k, l, i, j \in \mathbb{N}}\left|d_{k l i j}\right|<\infty .
$$

(ii) Assume that $1<p<\infty$. In that case, $D \in\left(\mathcal{L}_{p}: \mathcal{M}_{u}\right)$ iff

$$
M_{1}=\sup _{k, l \in \mathbb{N}} \sum_{i, j}\left|d_{k l i j}\right|^{p^{\prime}}<\infty .
$$

(iii) Assume that $0<p \leq 1$ and $1 \leq p_{1}<\infty$. In that case, $D \in\left(\mathcal{L}_{p}: \mathcal{L}_{p_{1}}\right)$ iff

$$
\sup _{i, j \in \mathbb{N}} \sum_{k, l}\left|d_{k l i j}\right|^{p_{1}}<\infty .
$$

(iv) Assume that $0<p \leq 1$. In that case, $D \in\left(\mathcal{L}_{p}: C_{b p}\right)$ iff the condition (3.1) holds and there exists a sequence $\left(\alpha_{i j}\right) \in \Omega$ such that

$$
b p-\lim _{k, l \rightarrow \infty} d_{k l i j}=\alpha_{i j} .
$$

(v) Assume that $1<p<\infty$. In that case, $D \in\left(\mathcal{L}_{p}: \mathcal{C}_{b p}\right)$ iff (3.2) and (3.3) hold.

Theorem 3.2. Consider the set $w_{1}$ defined by

$$
w_{1}=\left\{t=\left(t_{i j}\right) \in \Omega: \sup _{i, j \in \mathbb{N}} \sum_{k, l}\left|\left(\begin{array}{l}
k \\
i
\end{array}\right)\left(\begin{array}{l}
l \\
j
\end{array}\right) s^{k-l-i} r^{l-k-j}(r+s)^{i+j} t_{k l}\right|<\infty\right\} .
$$

Then, $\left(\mathcal{B}_{p}^{r, s}\right)^{\alpha}=w_{1}$ for $0<p \leq 1$.

Proof. Consider the 4-dimensional matrix $G^{r, s}=\left(g_{k l i j}^{r, s}\right)$ defined by

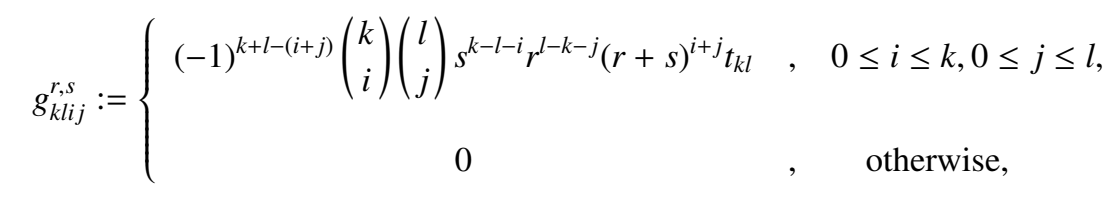

for every $k, l, i, j \in \mathbb{N}$. In that case, by using the relation (2.5) we obtain that

$$
\begin{aligned}
t_{k l} u_{k l} & =t_{k l} \sum_{i, j=0}^{k, l}(-1)^{k+l-(i+j)}\left(\begin{array}{l}
k \\
i
\end{array}\right)\left(\begin{array}{l}
l \\
j
\end{array}\right) s^{k-l-i} r^{l-k-j}(r+s)^{i+j} v_{i j} \\
& =\sum_{i, j=0}^{k, l}\left\{(-1)^{k+l-(i+j)}\left(\begin{array}{l}
k \\
i
\end{array}\right)\left(\begin{array}{l}
l \\
j
\end{array}\right) s^{k-l-i} r^{l-k-j}(r+s)^{i+j} t_{k l}\right\} v_{i j} \\
& =\left(G^{r, s} v\right)_{k l}
\end{aligned}
$$

for every $k, l \in \mathbb{N}$. In this fact, we conclude from relation (3.4) that $t u=\left(t_{k l} u_{k l}\right) \in \mathcal{L}_{u}$ whenever $u \in \mathcal{B}_{p}^{r, s}$ iff $G^{r, s} v \in \mathcal{L}_{u}$ whenever $v \in \mathcal{L}_{p}$. This means that $t=\left(t_{k l}\right) \in\left(\mathcal{B}_{p}^{r, s}\right)^{\alpha}$ iff $G^{r, s} \in\left(\mathcal{L}_{p}: \mathcal{L}_{u}\right)$. Then, we derive by using part (iii) of Lemma 3.1 with $p_{1}=1$ that

$$
\sup _{i, j \in \mathbb{N}} \sum_{k, l}\left|\left(\begin{array}{l}
k \\
i
\end{array}\right)\left(\begin{array}{l}
l \\
j
\end{array}\right) s^{k-l-i} r^{l-k-j}(r+s)^{i+j} t_{k l}\right|<\infty .
$$

This yields the desired consequence that $\left(\mathcal{B}_{p}^{r, s}\right)^{\alpha}=w_{1}$ for $0<p \leq 1$. 
Theorem 3.3. Consider the sets $w_{2}, w_{3}$ and $w_{4}$ defined by

$$
\begin{aligned}
& w_{2}=\left\{t=\left(t_{i j}\right) \in \Omega: \sup _{k, l, j, j \in \mathbb{N}}\left|\sum_{m=i}^{k} \sum_{n=j}^{l}\left(\begin{array}{c}
m \\
i
\end{array}\right)\left(\begin{array}{c}
n \\
j
\end{array}\right) s^{m-n-i} r^{n-m-j}(r+s)^{i+j} t_{m n}\right|<\infty\right\}, \\
& w_{3}=\left\{t=\left(t_{i j}\right) \in \Omega: b p-\lim _{k, l \rightarrow \infty} \sum_{m=i}^{k} \sum_{n=j}^{l}\left(\begin{array}{c}
m \\
i
\end{array}\right)\left(\begin{array}{c}
n \\
j
\end{array}\right)(-1)^{m+n-(i+j)} s^{m-n-i} r^{n-m-j}(r+s)^{i+j} t_{m n} \quad \text { exists }\right\}, \\
& w_{4}=\left\{t=\left(t_{i j}\right) \in \Omega: \sup _{k, l \in \mathbb{N}} \sum_{i, j}\left|\sum_{m=i}^{k} \sum_{n=j}^{l}\left(\begin{array}{c}
m \\
i
\end{array}\right)\left(\begin{array}{c}
n \\
j
\end{array}\right) s^{m-n-i} r^{n-m-j}(r+s)^{i+j} t_{m n}\right|^{p^{\prime}}<\infty\right\} .
\end{aligned}
$$

\section{In that case, following statements are satisfied:}

(i) Assume that $0<p \leq 1$. In that case, $\left(\mathcal{B}_{p}^{r, s}\right)^{\beta(b p)}=w_{2} \cap w_{3}$,

(ii) Assume that $1<p<\infty$. In that case, $\left(\mathcal{B}_{p}^{r, s}\right)^{\beta(b p)}=w_{3} \cap w_{4}$.

Proof. Let $t=\left(t_{i j}\right) \in \Omega$ and $u \in \mathcal{B}_{p}^{r, s}$ be given. Then, we can conclude from Theorem 2.4 that there exists a double sequence $v=\left(v_{i j}\right) \in \mathcal{L}_{p}$. Define the 4-dimensional matrix $O^{r, s}=\left(o_{k l i j}^{r, s}\right)$ by

$$
o_{k l i j}^{r, s}:=\left\{\begin{array}{cc}
\sum_{m=i}^{k} \sum_{n=j}^{l}(-1)^{k+l-(i+j)}\left(\begin{array}{c}
m \\
i
\end{array}\right)\left(\begin{array}{c}
n \\
j
\end{array}\right) s^{m-n-i} r^{n-m-j}(r+s)^{i+j} t_{m n} & 0 \leq i \leq k, 0 \leq j \leq l, \\
0 & , \quad \text { otherwise, }
\end{array}\right.
$$

for every $k, l, i, j \in \mathbb{N}$. Therefore, we obtain by the relation (2.5) that,

$$
\begin{aligned}
z_{k l} & =\sum_{i, j=0}^{k, l} t_{i j} u_{i j} \\
& =\sum_{i, j=0}^{k, l} t_{i j}\left\{\sum_{m, n=0}^{i, j}(-1)^{i+j-(m+n)}\left(\begin{array}{c}
i \\
m
\end{array}\right)\left(\begin{array}{l}
j \\
n
\end{array}\right) s^{i-j-m} r^{j-i-n}(r+s)^{m+n} v_{m n}\right\} \\
& =\sum_{i, j=0}^{k, l}\left\{\sum_{m=i}^{k} \sum_{n=j}^{l}(-1)^{m+n-(i+j)}\left(\begin{array}{c}
k \\
i
\end{array}\right)\left(\begin{array}{l}
l \\
j
\end{array}\right) s^{m-n-i} r^{n-m-j}(r+s)^{i+j} t_{m n}\right\} v_{i j} \\
& =\left(O^{r, s} v\right)_{k l}
\end{aligned}
$$

for every $k, l \in \mathbb{N}$. Then by considering the equality above, we deduce that $t u=\left(t_{k l} u_{k l}\right) \in C \mathcal{S}_{b p}$ whenever $u=\left(u_{k l}\right) \in$ $\mathcal{B}_{p}^{r, s}$ iff $z=\left(z_{k l}\right) \in C_{b p}$ whenever $v=\left(v_{k l}\right) \in \mathcal{L}_{p}$. This leads us to the fact that $t=\left(t_{k l}\right) \in\left(\mathcal{B}_{p}^{r, s}\right)^{\beta(b p)}$ iff $O^{r, s} \in\left(\mathcal{L}_{p}: C_{b p}\right)$. Hence;

(i) If $0<p \leq 1$, then from Part (iv) of Lemma 3.1, we achieve that $\left(\mathcal{B}_{p}^{r s}\right)^{\beta(b p)}=w_{2} \cap w_{3}$,

(ii) If $1<p<\infty$, then from Part (v) of Lemma 3.1, we have $\left(\mathcal{B}_{p}^{r, s}\right)^{\beta(b p)}=w_{3} \cap w_{4}$.

Theorem 3.4. (i) If $0<p \leq 1$, then $\left(\mathcal{B}_{p}^{r, s}\right)^{\gamma}=w_{2}$,

(ii) If $1<p<\infty$, then $\left(\mathcal{B}_{p}^{r, s}\right)^{\gamma}=w_{4}$.

Proof. This can be obtained by analogy with the proof of Theorem 3.3 with Parts (i) and (ii) of Lemma 3.1 instead of Parts (iv) and (v), respectively. Therefore, we leave the details.

\section{Some Matrix Transformations Related to the Sequence Space $\mathcal{B}_{p}^{r, s}$}

In this section, we give the characterization of the classes $\left(\mathcal{B}_{p}^{r, s}: \Lambda\right)$, where $\Lambda \in\left\{\mathcal{M}_{u}, \mathcal{C}_{b p}\right\}$ for the both cases $0<p \leq 1$ and $1<p<\infty$ and $\left(\mathcal{B}_{p}^{r, s}: \mathcal{L}_{q}\right)$ for $0<p \leq 1$ and $1 \leq q<\infty$. 
Theorem 4.1. Assume that $D=\left(d_{k l i j}\right)$ be an arbitrary 4-dimensional infinite matrix. In that case:

(i) If $0<p \leq 1$, then $D \in\left(\mathcal{B}_{p}^{r, s}: \mathcal{M}_{u}\right)$ iff

$$
D_{k l} \in\left\{\mathcal{B}_{p}^{r, s}\right\}^{\beta(\vartheta)}
$$

and

$$
\sup _{k, l, i, j \in \mathbb{N}}\left|\sum_{a=i}^{\infty} \sum_{b=j}^{\infty}\left(\begin{array}{c}
a \\
i
\end{array}\right)\left(\begin{array}{c}
b \\
j
\end{array}\right) s^{a-b-i} r^{b-a-j}(r+s)^{i+j} d_{k l a b}\right|<\infty .
$$

(ii) If $1<p<\infty$, then $D \in\left(\mathcal{B}_{p}^{r, s}: \mathcal{M}_{u}\right)$ iff the condition (4.1) holds and

$$
\sup _{k, l \in \mathbb{N}} \sum_{i, j}\left|\sum_{a=i}^{\infty} \sum_{b=j}^{\infty}\left(\begin{array}{l}
a \\
i
\end{array}\right)\left(\begin{array}{l}
b \\
j
\end{array}\right) s^{a-b-i} r^{b-a-j}(r+s)^{i+j} d_{k l a b}\right|^{p^{\prime}}<\infty .
$$

Proof. In the case of $0<p \leq 1$, the theorem can be proved by using similar method of the proof of the second part, we give the proof only for $1<p<\infty$.

(ii) Let $1<p<\infty$ and $D \in\left(\mathcal{B}_{p}^{r, s}: \mathcal{M}_{u}\right)$. Then, $D u$ exists and in $\mathcal{M}_{u}$ for every $u \in \mathcal{B}_{p}^{r, s}$ which implies the fact that $D_{k l} \in\left\{\mathcal{B}_{p}^{r, s}\right\}^{\beta(\vartheta)}$. By taking into account the equality $(2.5)$, the $(m, n)$ th rectangular partial sum of the series $\sum_{i, j} d_{k l i j} u_{i j}$ obtained as

$$
\begin{aligned}
(D u)_{k l}^{[m, n]} & =\sum_{i, j=0}^{m, n} d_{k l i j} u_{i j} \\
& =\sum_{i, j=0}^{m, n} d_{k l i j}\left[\sum_{a=0}^{i} \sum_{b=0}^{j}(-1)^{i+j-(a+b)}\left(\begin{array}{l}
i \\
a
\end{array}\right)\left(\begin{array}{c}
j \\
b
\end{array}\right) s^{i-j-a} r^{j-i-b}(r+s)^{a+b} v_{a b}\right] \\
& =\sum_{i, j=0}^{m, n}\left[\sum_{a=i}^{m} \sum_{b=j}^{n}(-1)^{a+b-(i+j)}\left(\begin{array}{c}
a \\
i
\end{array}\right)\left(\begin{array}{l}
b \\
j
\end{array}\right) s^{a-b-i} r^{b-a-j}(r+s)^{i+j} d_{k l a b}\right] v_{i j}
\end{aligned}
$$

for all $k, l, m, n \in \mathbb{N}$. Let us define the 4-dimensional matrix $H=\left(h_{k l i j}\right)$ as $h_{k l i j}:=$

$$
\left\{\begin{array}{cc}
\sum_{a=i}^{\infty} \sum_{b=j}^{\infty}(-1)^{a+b-(i+j)}\left(\begin{array}{c}
a \\
i
\end{array}\right)\left(\begin{array}{c}
b \\
j
\end{array}\right) s^{a-b-i} r^{b-a-j}(r+s)^{i+j} d_{k l a b}, & 0 \leq k \leq i, 0 \leq l \leq j, \\
0 & \text { otherwise }
\end{array}\right.
$$

for all $k, l, i, j \in \mathbb{N}$. Then, by taking $\vartheta$-limit on (4.4) while $m, n \rightarrow \infty$, we have that $D u=H v$. So, if we take into account the fact that $D=\left(d_{k l i j}\right) \in\left(\mathcal{B}_{p}^{r, s}: \mathcal{M}_{u}\right)$ if and only if $H \in\left(\mathcal{L}_{p}: \mathcal{M}_{u}\right)$ with Part (ii) of Lemma 3.1, then it is obvious that the condition (4.3) holds.

Conversely, suppose that the conditions (4.1) and (4.3) hold. Let us choose the sequence $u \in \mathcal{B}_{p}^{r, s}$ with $v \in \mathcal{L}_{p}$ from the relation (2.2). Since, the condition (4.1) holds, then $D u$ exists. By using the relation (2.5), one can derive from the $(\varpi, \varsigma)$ th rectangular partial sum of the series $\sum_{i, j} d_{k l i j} u_{i j}$ for all $k, l, \varpi, \varsigma$ that

$$
\sum_{i, j=0}^{\varpi, \varsigma} d_{k l i j} u_{i j}=\sum_{i, j=0}^{\varpi, \varsigma}\left[\sum_{a=i}^{\varpi} \sum_{b=j}^{\varsigma}(-1)^{a+b-(i+j)}\left(\begin{array}{c}
a \\
i
\end{array}\right)\left(\begin{array}{l}
b \\
j
\end{array}\right) s^{a-b-i} r^{b-a-j}(r+s)^{i+j} d_{k l a b}\right] v_{i j} .
$$

By taking $\vartheta$-limit in the equality above as $\varpi, \varsigma \rightarrow \infty$, it can be easily obtain from the following equality for every $k, l \in \mathbb{N}$

$$
\sum_{i, j} d_{k l i j} u_{i j}=\sum_{i, j} h_{k l i j} v_{i j}
$$

that $D u=H v$. From the condition (4.3), it is known that $H \in\left(\mathcal{L}_{p}: \mathcal{M}_{u}\right)$ and thus it is obvious that $D \in\left(\mathcal{B}_{p}^{r, s}: \mathcal{M}_{u}\right)$, as desired.

To avoid the repetition of the similar statements, we give the following two theorems without proof since they may be proved in the similar way used in proving Theorem 4.1. 
Theorem 4.2. Let $D=\left(d_{k l i j}\right)$ be any 4-dimensional matrix. In that case, following statements are hold:

(i) Let $0<p \leq 1$. Then, $D \in\left(\mathcal{B}_{p}^{r, s}: C_{b p}\right)$ iff the conditions (4.1) and (4.2) hold and there exists $\left(\alpha_{i j}\right) \in \Omega$ such that

$$
b p-\lim _{k, l \rightarrow \infty} \sum_{a=i}^{\infty} \sum_{b=j}^{\infty}(-1)^{a+b-i+j}\left(\begin{array}{l}
a \\
i
\end{array}\right)\left(\begin{array}{l}
b \\
j
\end{array}\right) s^{a-b-i} r^{b-a-j}(r+s)^{i+j} d_{k l a b}=\alpha_{i j},
$$

(ii) Let $1<p<\infty$. Then, $D \in\left(\mathcal{B}_{p}^{r, s}: \mathcal{C}_{b p}\right)$ iff the conditions (4.1), (4.3) and (4.5) hold.

Theorem 4.3. Let $0<p \leq 1,1 \leq q<\infty$ and $D=\left(d_{k l i j}\right)$ be any 4-dimensional matrix. Then, $D \in\left(\mathcal{B}_{p}^{r, s}: \mathcal{L}_{q}\right)$ iff the condition (4.1) holds and

$$
\sup _{k, l \in \mathbb{N}} \sum_{i, j}\left|\sum_{a=i}^{\infty} \sum_{b=j}^{\infty}\left(\begin{array}{l}
a \\
i
\end{array}\right)\left(\begin{array}{l}
b \\
j
\end{array}\right) s^{a-b-i} r^{b-a-j}(r+s)^{i+j} d_{k l a b}\right|^{q}<\infty .
$$

ACKNOWLEDGEMENT

The authors would like to express their gratitude to the referees for their very valuable comments and suggestions.

\section{CONFLICTS OF INTEREST}

The authors declare that there are no conflicts of interest regarding the publication of this article.

\section{REFERENCES}

[1] Altay, B., Başar, F., Some new spaces of double sequences, J. Math. Anal. Appl., 309(1)(2005), 70-90. 1

[2] Bașar, F., Summability Theory and Its Applications, Bentham Science Publishers, e-book, Monographs, Istanbul, 2012. 1

[3] Başar, F., Sever, Y., The space $\mathcal{L}_{q}$ of double sequences, Math. J. Okayama Univ., 51(2009), 149-157. 1, 2, 2

[4] Bişgin, M.C., The binomial sequence spaces which include the spaces $\ell_{p}$ and $\ell_{\infty}$ and geometric properties, Journal of Inequalities and Applications (2016):304. 1

[5] Boss, J., Classical and Modern Methods in Summability, Oxford University Press, Newyork, 2000. 2, 2.5, 2.8

[6] Çapan, H., Bașar, F., Some paranormed difference spaces of double sequences, Indian L. Math., 58(3)(2016),405-427. 1

[7] Demiriz, S., Duyar, O., Domain of the Cesàro mean matrix in some paranormed spaces of double sequences, Contemp. Anal. Appl. Math., 3(2)(2015), 247-262. 1

[8] Demiriz, S., Erdem, S., Domain of Euler-Totient Matrix Operator in the Space $\mathcal{L}_{p}$, Korean J. Math., 28(2)(2020), 361-378. 1

[9] Hamilton, H. J., Transformations of multiple sequences, Duke Math. J., 2(1936), 29-60. 2.1, 2.2

[10] Mòricz, F., Extensions of the spaces $c$ and $c_{0}$ from single to double sequences, Acta Math. Hungar., 57(1991), 129-136. 1

[11] Mursaleen, M., Almost strongly regular matrices and a core theorem for double sequences, J. Math. Anal. Appl., 293(2)(2004), 523-531. 1

[12] Robison, G. M., Divergent double sequences and series, Amer. Math. Soc. Trans., 28(1926), 50-73. 2.1, 2.2

[13] Schaefer, H.H., Topological Vector Spaces, Graduate Texts in Matematics, Volume 3, 5th printing, 1986. 2.6

[14] Talebi, G., Operator norms of four-dimensional Hausdorff matrices on the double Euler sequence spaces, Linear and Multilinear Algebra, 65(11)(2017), 2257-2267. 1

[15] Tug̃, O., Başar, F., Four-Dimensional Generalized Difference Matrix and Some Double Sequence Spaces, AIP Conference Proceedings, AIP Publishing LLC, 1759(1)(2016), p.020075. 1

[16] Tug̃, O., Four-dimensional generalized difference matrix and some double sequence spaces, J. Inequal. Appl. (1)(2017), 149. 1

[17] Tug̃, O., Rakočević, V., Malkowsky, E., On the Domain of the Four-Dimensional Sequential Band Matrix in Some Double Sequence Spaces, Mathematics 789(8)(2020), doi:10.3390/math8050789. 1

[18] Yeşilkayagil, M., Başar, F., Domain of Riesz mean in the space $\mathcal{L}_{s}$, Filomat, 31(4)(2017), 925-940. 1, 2, 3.1

[19] Yeşilkayagil, M., Başar, F., Domain of Riesz mean in some spaces of double sequences, Indagationes Mathematicae, 29(2018), 1009-1029. 1

[20] Yeşilkayagil, M., Başar, F., Domain of Euler Mean in the Space of Absolutely p-Summable Double Sequences with $0<p<1$, Anal. Theory Appl., 34(3)(2018), 241-252. 1

[21] Zeltser, M., Investigation of double sequence spaces by soft and hard analitic methods, Dissertationes Mathematicae Universtaties Tartuensis 25, Tartu University Press, Univ. of Tartu, Faculty of Mathematics and Computer Science, Tartu, 2001. 1, 2

[22] Zeltser, M., On conservative matrix methods for double sequence spaces, Acta Math. Hung., 95(3)(2002), 225-242. 1 